\title{
Cirurgia Bariátrica Cura Síndrome Metabólica?
}

\begin{abstract}
RESUMO
Objetivos: Avaliar o impacto da cirurgia bariátrica na síndrome metabólica (SM) e quais os critérios que mais contribuíram para sua remissão após cirurgia. A evolução da leucometria também foi analisada. Material e método: 47 mulheres obesas com SM foram avaliadas. Todas as pacientes foram operadas pela técnica da gastroplastia vertical em Y-de-Roux, com colocação de anel de contenção na anastomose gastro-jejunal (Fobi-Capella). Pacientes foram avaliadas antes da cirurgia e no primeiro ano pós-operatório. Resultados: $A$ glicemia de jejum apresentou queda relevante nos 3 primeiros meses. Ao final de 12 meses, todas as 20 pacientes que tinham DM2 ou glicemia de jejum alterada apresentavam níveis glicêmicos e hemoglobina glicosilada normais. Nenhuma delas estava usando drogas anti-diabéticas. Valores de triglicerídeos reduziram 49,2\%. O HDL-colesterol aumentou $27,2 \%$. A redução da pressão arterial foi, em média, de $28,7 \mathrm{mmHg}$ na sistólica e de 20,8 na diastólica. A contagem de leucócitos caiu de $7671 / \mu \mathrm{L}$ para $6156 / \mu \mathrm{L}$. Glicemia de jejum, triglicerídeos, pressão diastólica e sistólica e HDL-colesterol foram as variáveis que mais contribuíram para a extinção da SM. No final do primeiro ano, essa extinção ocorreu em $80,9 \%$ das pacientes. Discussão: Cirurgia bariátrica reduz a resistência à insulina e conseqüentemente os riscos cardiovasculares. (Arq Bras Endocrinol Metab 2007;51/1:79-85)
\end{abstract}

Descritores: Diabetes mellitus tipo 2; Perda de peso; Hipertensão; Dislipidemia; Obesidade; Bypass gástrico; Fatores de risco cardiovascular

\section{ABSTRACT}

\section{Can Bariatric Surgery Cure Metabolic Syndrome?}

Objectives: To evaluate the impact of bariatric surgery on the metabolic syndrome (MS) and what are the criteria that contribute the most for its exclusion after surgery. The progress of leucometry was also analyzed. Methods and procedures: 47 obese women with MS were evaluated. All patients were operated with the Roux-en-Y vertical gastric bypass technique, with the insertion of a contention ring on the gastro-jejune anastomosis (Fobi-Capella). Patients were evaluated before and on the first year post-surgery. Results: Fasting glucose presented a relevant decrease at 3 months after surgery. After 12 months, all 20 patients who had DM2 or altered fasting glucose presented normal levels of fasting glucose and glicated hemoglobin, and none of them was using any anti-diabetic drug. Triglycerides levels were reduced by $49.2 \%$, whereas HDL-cholesterol increased by $27.2 \%$. Systolic and diastolic blood pressures were reduced by an average of 28.7 and $20.8 \mathrm{mmHg}$, respectively. Leucocytes counting fell from $7671 / \mu \mathrm{L}$ to $6156 / \mu \mathrm{L}$. Fasting glucose, triglycerides, DBP, SBP and HDL-cholesterol were the variables that contributed most for the reduction of MS. At the end of the first year, elimination of MS occurred in $80.9 \%$ of the patients. Discussion: Bariatric surgery reduces resistance to insulin and consequently the cardiovascular risk factors. (Arq Bras Endocrinol Metab 2007;51/1:79-85)

Keywords: Type 2 diabetes mellitus; Weight loss; Hypertension; Dyslipidemia; Obesity; Gastric bypass; Cardiovascular risk factors

\section{artigo original}

\author{
Perseu Seixas de Carvalho \\ Cora LAVIGNe DE C.B. Moreira \\ MELINA DA COSTA BARELLI \\ flávia Heringer de Oliveira \\ MARIANA FURIERI GUZZO \\ Gustavo P. SoAREs Miguel \\ ELIANA ZANDONADE
}

Serviço de Endocrinologia do Hospital Universitário Cassiano

Antônio Moraes (PSC, CLCBM,

$\mathrm{MCB}, \mathrm{FHO} \& \mathrm{MFG}) \mathrm{e}$

Departamento de Clínica

Cirúrgica (GPSM), Centro

Biomédico, e Departamento de

Estatística (EZ), Centro de Ciências

Exatas, Universidade Federal do

Espírito Santo, Vitória, ES.

Recebido em 25/04/05

Aceito em 26/07/06 
$A^{s}$ Síndrome Metabólica (SM) é caracterizada por uma diminuição, genética e/ou adquirida, da sensibilidade à insulina, principalmente em músculo, fígado e tecido adiposo. Isso determina uma elevação dos níveis glicêmicos e leva a uma hiperinsulinemia compensatória que predispõe ao desenvolvimento de doença vascular aterosclerótica, síndrome do ovário policístico, esteato-hepatite não alcoólica e possivelmente diversas formas de câncer (1). Além disso, a SM está associada a um estado pró-inflamatório crônico, que pode ser avaliado pelo aumento de uma série de marcadores, incluindo a contagem de leucócitos, concentrações de proteína $\mathrm{C}$ reativa (PCR), interleucinas e inibidor do ativador do plasminogênio 1 (PAI l) $(2,3)$.

A obesidade mórbida, um estado de insulinoresistência por excelência (4), está freqüentemente associada à SM, que aumenta a mortalidade geral em cerca de 1,5 vezes e a cardiovascular em cerca de 2,5 vezes (5-9). A prevalência da SM em obesos mórbidos, na maioria dos estudos, encontra-se acima de 50\% (10). Em um levantamento feito numa população de 249 obesos mórbidos do ambulatório de obesidade do nosso serviço, Hospital Universitário Cassiano Antônio Moraes - HUCAM, a prevalência de SM foi de 73\% (dados não publicados).

Existem evidências de que perdas de peso moderadas, entre $5 \%$ a $10 \%$ do peso inicial, com tratamentos convencionais, promovem benefícios metabólicos (11). Entretanto, estes tratamentos têm sido pouco efetivos em obesos mórbidos e não se mostram sustentáveis no longo prazo (12).

A cirurgia bariátrica é considerada o tratamento mais radical para a obesidade, porém é o único que resulta em perda de peso expressiva $(20 \%$ a $40 \%$ do peso inicial) e mantida por, pelo menos, 15 anos (1315), determinando melhora dos parâmetros metabóli$\cos (16,17)$. É um tratamento reservado para pacientes com IMC $\geq 40 \mathrm{~kg} / \mathrm{m}^{2}$ ou IMC $\geq 35 \mathrm{~kg} / \mathrm{m}^{2}$ com comorbidades associadas $(18,19)$ e com tratamentos convencionais prévios mal sucedidos.

O objetivo deste trabalho é avaliar o impacto da cirurgia bariátrica sobre a Síndrome Metabólica e quais os critérios que mais contribuem para a sua remissão após a cirurgia. Analisamos também a evolução da leucometria como marcadora do estado inflamatório crônico subclínico $(6,7,20-23)$ nas pacientes estudadas.

\section{MATERIAL E MÉTODOS}

Foram avaliadas 47 mulheres obesas, duas com obesidade grau II (IMC 35-39,9 kg/m²) e quarenta e cinco com obesidade grau III (IMC $\geq 40 \mathrm{~kg} / \mathrm{m}^{2}$ ) (24), todas com o diagnóstico de SM, com idade média de 41,5 anos $\pm 10,4$
Tabela 1. Componentes da síndrome metabólica segundo o NCEP ATP III.

\begin{tabular}{lcc}
\hline & Homens & Mulheres \\
Circunferência abdominal $(\mathrm{cm})$ & $\geq 102$ & $\geq 88$ \\
HDL-colesterol $(\mathrm{mg} / \mathrm{dL})$ & $\leq 40$ & $\leq 50$ \\
Triglicerídeos $(\mathrm{mg} / \mathrm{dL})$ & $\geq 150$ & $\geq 150$ \\
Glicemia de jejum $(\mathrm{mg} / \mathrm{dL})$ & $\geq 110$ & $\geq 110$ \\
Pressão arterial $(\mathrm{mmHg})$ & $\geq 130 / 85$ & $\geq 130 / 85$ \\
\hline
\end{tabular}

(20-65 anos), com média de IMC de 49,4 \pm 7,3 (36,9-71,5), admitidas no Ambulatório de Obesidade do HUCAM da Universidade Federal do Espírito Santo, e submetidas à cirurgia bariátrica no período de 5 de junho de 2002 até 4 de agosto de 2004, pela equipe de cirurgia bariátrica deste hospital. As pacientes apresentavam um risco cirúrgico compatível com a cirurgia proposta, avaliação psicológica favorável e foram previamente informadas dos possíveis riscos e benefícios do procedimento. Todas as pacientes foram operadas pela técnica da gastroplastia vertical em Y-de-Roux (GVYR), com colocação de um anel de contensão na anastomose gastro-jejunal (FobiCapella) (25). O diagnóstico pré-operatório de SM foi firmado com base nos critérios do NCEP-ATP III, que considera como portadores da SM indivíduos que apresentem pelo menos três dos cinco critérios descritos na tabela 1 (8).

A circunferência abdominal foi medida com a paciente em pé, em nível da linha média entre o rebordo costal e a crista ilíaca superior. Todos os exames laboratoriais foram realizados no Laboratório de Análises Clínicas do HUCAM utilizando-se o sistema de química clínica Dimension - Dade Behring, Inc. Os hemogramas foram realizados pelo equipamento Coulter $\mathrm{T}$ 890 e a contagem diferencial dos leucócitos obtida após coloração pelo método May Grunwald - Giemsa. A pressão arterial (PA) foi aferida com a paciente sentada, após mais de 5 minutos de repouso, por pelo menos duas vezes durante a consulta, sendo considerada sempre a menor medida. Foram consideradas hipertensas pacientes que estivessem em uso de antihipertensivos, independente do nível de PA obtido nas medidas.

As pacientes foram avaliadas antes da cirurgia e no primeiro mês de pós-operatório, e, em seguida, a cada três meses durante o primeiro ano, desde que não apresentassem complicações que justificassem intervalos menores. Foram registrados, a cada consulta, dados de exames físico e laboratoriais que permitiram analisar os critérios para diagnóstico da SM. Nas consultas intermediárias, nem todas as pacientes que fizeram parte do estudo compareceram; assim, os resultados intermediários são apresentados, embora com um número menor de pacientes, mas a análise final, de doze meses, foi feita com todas as 47 pacientas iniciais.

\section{RESULTADOS}

As características antes e depois da cirurgia foram comparadas por teste $t$ de Student pareado e bi-caudal. As diferenças entre proporções foram analisadas pelo teste de McNemar para medir a diferença entre as situações 
Tabela 2. Dados antropométricos e variáveis metabólicas.

\begin{tabular}{lc}
\hline Variáveis & Pré-operatório \\
№ $(\%)$ de pacientes com SM & $47(100 \%)$ \\
Idade (anos) & $41,5 \pm 10,4(20-65)$ \\
IMC $\left(\mathrm{kg} / \mathrm{m}^{2}\right)$ & $49,4 \pm 7,3(36,9-71,5)$ \\
PA sistólica $(\mathrm{mmHg})$ & $150,9 \pm 21,6$ \\
PA diastólica $(\mathrm{mmHg})$ & $99,8 \pm 15,2$ \\
Circunferência abdominal $(\mathrm{cm})$ & $130 \pm 13,1$ \\
Glicemia de jejum $(\mathrm{mg} / \mathrm{dL})$ & $119 \pm 46,6$ \\
HDL-colesterol $(\mathrm{mg} / \mathrm{dL})$ & $44,4 \pm 10,2$ \\
Triglicerídeos $(\mathrm{mg} / \mathrm{dL})$ & $174,5 \pm 84,3$ \\
Leucócitos $(\mathrm{n}$ o $\mu \mathrm{L})$ & $7670,7 \pm 2070,3$ \\
\hline
\end{tabular}

de presença de SM antes da cirurgia e aos três, seis, nove e doze meses. As análises estatísticas foram realizadas usando o programa SPSS 11.5 (SPSS Inc. Chicago, IL). Um $\mathrm{p}<0,05$ foi considerado estatisticamente significante para todas as análises. Os dados antropométricos e parâmetros bioquímicos da população estudada estão listados na tabela 2.

Das 47 pacientes analisadas, todas apresentavam, antes da cirurgia, pelo menos três dos cinco critérios listados na tabela 1 e foram classificadas como tendo SM. Dezoito pacientes (38\%) apresentavam quatro critérios, e em seis pacientes (12\%) todos os cinco critérios estavam presentes.

Dos cinco parâmetros utilizados para diagnóstico da SM, quatro deles (glicemia de jejum, triglicerídeos, pressão arterial sistêmica e HDL-colesterol) apresentaram, após 12 meses de cirurgia, modificações que determinaram valores médios que os excluíam do diagnóstico de SM (tabelas 1 e 3), contribuindo, assim, para a remissão da SM (figuras 1 e 8).

As variáveis que mais contribuíram para a remissão da SM foram, nesta ordem: glicemia de jejum, triglicerídeos, PAD, PAS e HDL colesterol.
Ao final do primeiro ano, a remissão da SM ocorreu em 38 pacientes $(80,9 \%)$ de 47 avaliadas $(\mathrm{p}=0,000)$ (figura 1) e o IMC apresentou uma redução de 49,4 $\mathrm{kg} / \mathrm{m}^{2}$ para $32,9 \mathrm{~kg} / \mathrm{m}^{2}(\mathrm{p}<0,0001)$ (figura 2 , tabela 3$)$.

A glicemia de jejum apresentou queda considerável aos três meses de pós-operatório, de 119,0 $\mathrm{mg} / \mathrm{dL}$ para $94,6 \mathrm{mg} / \mathrm{dL}$; ao final dos 12 meses de avaliação, o valor da glicemia encontrava-se em 86,2 $\mathrm{mg} / \mathrm{dL}(\mathrm{p}=0,00001)$ (figura 6 , tabela 3 ).

No pré-operatório, 15 pacientes do grupo estudado $(31,9 \%)$ apresentavam diagnóstico de diabetes mellitus tipo 2 (DM 2) e 5 pacientes apresentavam glicemia de jejum alterada (acima de $110 \mathrm{mg} / \mathrm{dL}$ ). Nove pacientes faziam uso de antidiabéticos orais (metformina ou metformina + sulfoniluréia) e uma paciente fazia uso de insulina. A duração do diabetes variava de 7 a 168 meses, com tempo médio de doença de 33,6 meses. Após 12 meses, as 20 pacientes apresentaram níveis normais de glicemia de jejum e hemoglobina glicosilada e nenhuma mais fazia uso de qualquer medicação antidiabética.

Os níveis de triglicerídeos apresentaram queda de $49,2 \%$ em comparação aos valores iniciais, atingindo uma média de $88,6 \mathrm{mg} / \mathrm{dL}$ ao final do estudo (figura 4 , tabela 3 ).

Os valores de HDL-colesterol apresentaram um aumento de $27,2 \%$ em relação aos valores do préoperatório, atingindo uma média de $53,4 \mathrm{mg} / \mathrm{dL}$. (figura 5 , tabela 3 ).

A redução da pressão arterial foi, em média, de $28,7 \mathrm{mmHg}$ para a PAS e de $20,8 \mathrm{mmHg}$ para a PAD, $19,0 \%$ e $20,8 \%$ respectivamente (figura 3 , tabela 3 ).

A medida da circunferência abdominal atingiu, ao final de um ano, uma média de $99,5 \mathrm{~cm}(\mathrm{p}=$ 0,00000 ) (tabela 3). Apesar da significância estatística da redução da medida da circunferência abdominal, ela ainda permaneceu acima do valor proposto pelo

Tabela 3. Evolução dos parâmetros metabólicos avaliados durante o pré- e pós-operatório*.

\begin{tabular}{|c|c|c|c|c|}
\hline Variáveis & Pré-operatório & 6 meses de PO & 12 meses de PO & $\mathbf{p}^{t}$ \\
\hline № (\%) de pacientes avaliados & $47(100 \%)$ & $36(76,6 \%)$ & $47(100 \%)$ & \\
\hline № (\%) de pacientes com SM & $47(100 \%)$ & $18(50 \%)$ & $9(19,1 \%)$ & $<0,0001$ \\
\hline IMC $\left(\mathrm{kg} / \mathrm{m}^{2}\right)$ & $49,4 \pm 7,4$ & $36,9 \pm 5,3$ & $32,9 \pm 4,98$ & $<0,0001$ \\
\hline PA sistólica (mmHg) & $150,9 \pm 21,6$ & $124,7 \pm 13,4$ & $122,2 \pm 16,9$ & $<0,0001$ \\
\hline PA diastólica (mmHg) & $99,8 \pm 15,2$ & $82,8 \pm 9,5$ & $79,0 \pm 12,0$ & $<0,0001$ \\
\hline HDL-colesterol (mg/dL) & $44,4 \pm 10,2$ & $44,3 \pm 12,3$ & $53,4 \pm 16,5$ & $<0,0019$ \\
\hline Triglicerídeos (mg/dL) & $174,5 \pm 84,3$ & $111,4 \pm 56,9$ & $88,6 \pm 40,7$ & $<0,0001$ \\
\hline Glicemia de jejum (mg/dL) & $119 \pm 46,6$ & $85,2 \pm 18,5$ & $86,2 \pm 10,2$ & $<0,0001$ \\
\hline Circunferência abdominal (cm) & $130 \pm 13,1$ & $111,4 \pm 12,7$ & $99,5 \pm 9,5$ & $<0,0001$ \\
\hline Leucócitos (no/ $\mu \mathrm{L})$ & $7670,7 \pm 2070,3$ & $7133,1 \pm 2816,6$ & $6155,5 \pm 1959,2$ & $<0,0001$ \\
\hline
\end{tabular}

* Valores expressos em média \pm DP a menos que seja indicado de outra maneira.

† Valor de p obtido ao comparar as variáveis pré-operatórias e após 12 meses de pós-operatório. Foram omitidos os dados de

3 e 9 meses, para simplificação e melhor visualização dos resultados. Estes dados são mostrados nas figuras 1, 2 e 3. 


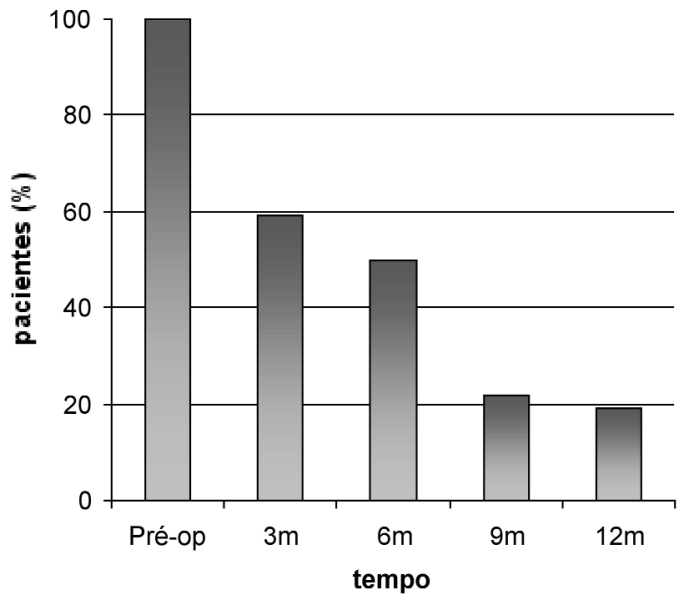

Figura 1. Prevalência da SM ao longo da avaliação.

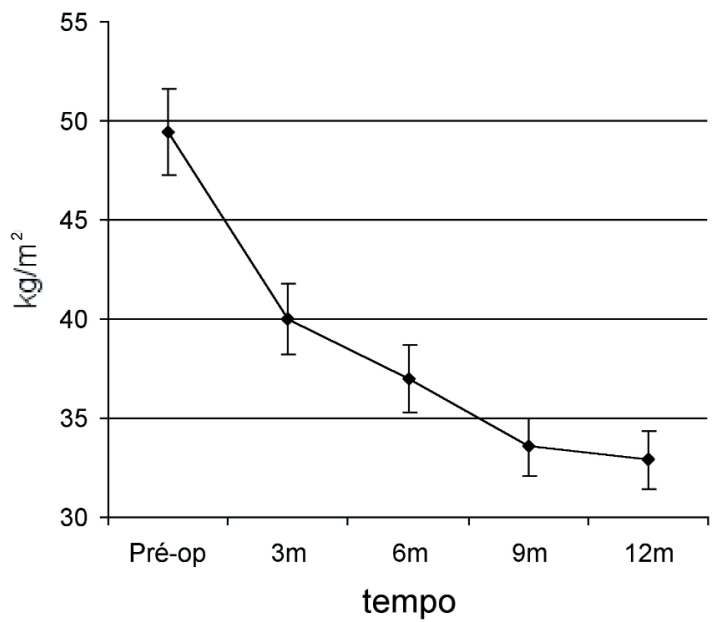

Figura 2. IMC.

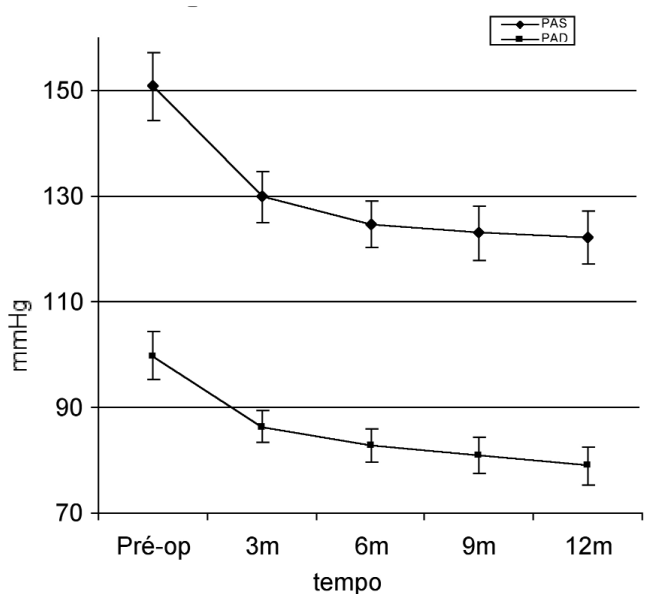

Figura 3. PAS e PAD.

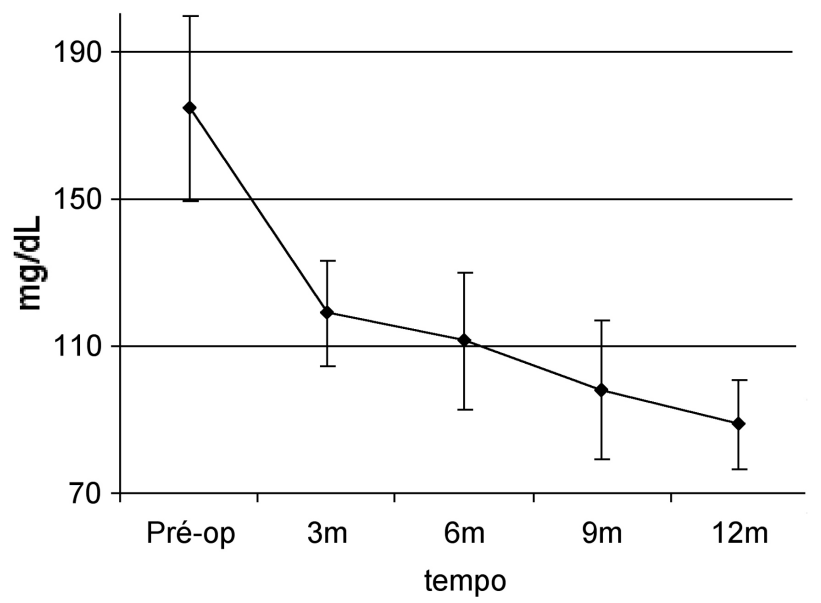

Figura 4. Triglicerídeos.

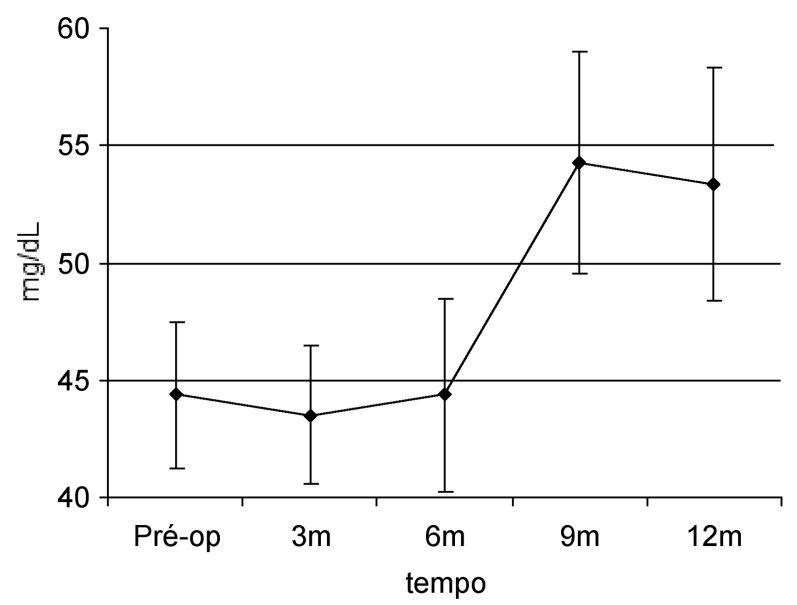

Figura 5. HDL-colesterol.

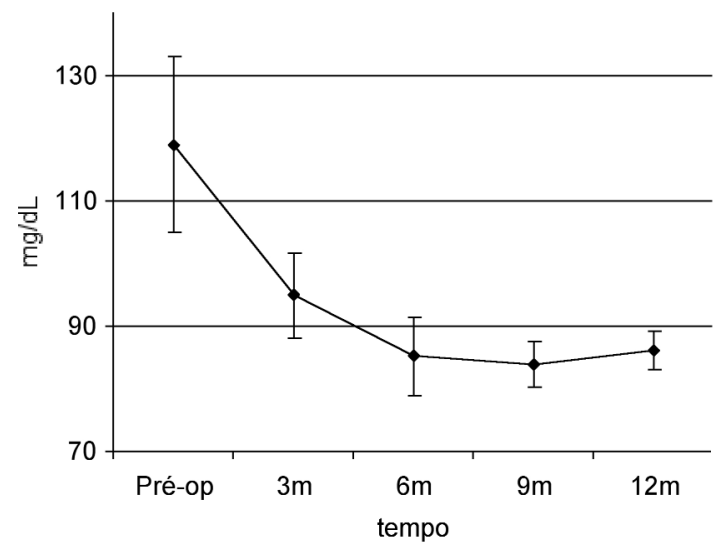

Figura 6. Glicemia de jejum. 
NCEP-ATP III como critério para diagnóstico da SM, sendo o parâmetro que menos contribuiu para a remissão no grupo estudado (figura 8).

A contagem dos leucócitos caiu de $7670,7 / \mu \mathrm{L}$ para $6155,5 / \mu \mathrm{L}$ ao final do estudo $(\mathrm{p}=0,00002)$ (figura 7 , tabela 3 ).

Durante os 12 meses de pós-operatório, a grande maioria dos pacientes pode descontinuar o uso de anti-hipertensivos e hipolipemiantes.

Nas pacientes avaliadas neste estudo, o índice de mortalidade foi de $0 \%$. Dez $(21,3 \%)$ pacientes apresentaram complicações pós-operatórias. Três $(6,38 \%)$ delas apresentaram deiscência de sutura de pele, 4 $(8,51 \%)$ hérnias incisionais, l $(2,2 \%)$ infecção de parede, $1(2,2 \%)$ estenose da anastomose gastrojejunal, $1(2,2 \%)$ empiema. As complicações tardias mais observadas foram queda de cabelo, deficiência de ferro, anemia e hipoproteinemia, todas solucionadas com a reposição oral de ferro, albumina e vitaminas.

\section{DISCUSSÃo}

A perda de peso que se segue à cirurgia bariátrica é acompanhada por uma melhora acentuada de todas as co-morbidades relacionadas à obesidade, incluindo apnéia do sono, refluxo gastro-esofágico, artropatias, infertilidade, estase venosa e úlceras por insuficiência venosa crônica $(24,26)$.

Neste estudo, o distúrbio do metabolismo glicídico foi o componente da SM que apresentou a mais significativa melhora. Assim, a GVYR se mostrou um método eficaz de reversão do DM tipo 2. Em cinco estudos publicados, em que foram avaliados 3.568 pacientes submetidos a GVYR, entre 82 e $98 \%$ dos pacientes diabéticos experimentaram completa remissão da doença. Nos mesmos estudos, a remissão da intolerância à glicose, sem DM 2, atingiu quase $100 \%(27-31)$.

Uma explicação simples para a melhora do metabolismo glicídico seria a imediata e severa privação de nutrientes que ocorre após a cirurgia. Esse estado levaria a uma redução brusca dos níveis de glicemia, diminuindo assim a glicotoxicidade sobre as células pancreáticas. Alguns dias depois, quando os pacientes retomam a ingestão alimentar, já apresentam perda de peso e um balanço energético negativo, condições que classicamente melhoram a tolerância à glicose.

Um outro mecanismo que pode agir em associação com o anterior seria um conjunto de alterações favoráveis na liberação de hormônios gastro-intesti- nais, que ocorrem após a GVYR, como redução nos níveis plasmáticos de ghrelina (32) e elevação dos níveis séricos de GLP-1 (33).

$\mathrm{Na} \mathrm{SM}$, a resistência à insulina no tecido adiposo determina uma intensa mobilização de AGL (34), substrato para a síntese de triglicerídeos no fígado, onde ocorre aumento da produção das VLDL, principais carreadoras dos mesmos $(35,36)$. Por outro lado, o aumento nos níveis plasmáticos de VLDLTGD provoca redução do HDL colesterol (37). Dessa forma, a redução dos triglicerídeos e o aumento significativo do HDL colesterol nas pacientes deste estudo traduzem uma melhora da sensibilidade à insulina tanto no fígado como no tecido adiposo.

Existem evidências de que pacientes hipertensos são em geral insulino-resistentes e hiperinsulinêmicos, quando comparados com indivíduos normotensos $(38,39)$. A retenção renal de sódio $(40)$, o aumento do tônus simpático central (41) e a diminuição da capacidade da insulina de induzir a produção de óxido nítrico pelo endotélio (42) são situações comuns na SM e são alguns dos mecanismos aos quais se atribui a ocorrência da hipertensão arterial. A perda de peso é acompanhada de melhora da hipertensão arterial, tanto em indivíduos com hipertensão idiopática como em portadores de SM.

A significativa redução na taxa global de leucócitos observada neste estudo sugere que a importante perda ponderal após a cirurgia pode reverter o estado pró-inflamatório crônico determinado pela SM $(43,44)$ e reduzir o risco de morbidade e mortalidade nesses indivíduos.

Após cerca de 55 anos de cirurgia bariátrica, as técnicas cirúrgicas foram expressivamente melhoradas, e a morbidade e mortalidade alcançaram níveis aceitáveis $(45)$ e bem menores quando comparados à manutenção em longo prazo do estado de obesidade (46), principalmente quando esta é associada à Síndrome Metabólica.

\section{CONCLUSÃo}

A cirurgia bariátrica, ao induzir significante perda ponderal em indivíduos obesos, diminui a resistência à insulina e, conseqüentemente, os fatores de risco cardiovasculares. Assim, pode ser considerada uma terapêutica efetiva para pacientes com SM e obesidade. Entretanto, neste estudo, a indicação para a cirurgia bariátrica não foi a presença da Síndrome Metabólica, e sim a presença de IMC $\geq 40 \mathrm{~kg} / \mathrm{m}^{2}$ ou IMC acima de $35 \mathrm{~kg} / \mathrm{m}^{2}$ com co-morbidades associadas. Desta forma, são necessários mais estudos para avaliar a efetividade da cirurgia bariátrica em indivíduos porta- 
dores de SM que apresentem obesidade com IMC abaixo de $35 \mathrm{~kg} / \mathrm{m}^{2}$, para se estabelecer novos pontos de corte na indicação deste procedimento.

\section{REFERÊNCIAS}

1. Reaven GM. Insulin resistance and its consequences. In: LeRoith D, Olefsky JM, Taylor SI, eds. Diabetes mellitus: A fundamental and clinical text. Bethesda: Lippincott Williams \& Wilkins, 2004. pp. 899-915.

2. Festa A, D'Agostino R Jr, Howard G, Mykkanen L, Tracy RP, Haffner SM. Chronic subclinical inflammation as part of the insulin resistance syndrome (IRAS). Circulation 2000;102 (1):42-7.

3. Vozarova B, Weyer C, Lindsay RS, Pratley RE, Bogardus C, Tataranni PA. High white blood cell count is associated with a worsening of insulin sensitivity and predicts the development of type 2 diabetes. Diabetes 2002;51(2):455-61.

4. Ferranini E, Natali A, Bell P, Cavallo-Perin P, Lalic N, Mingrone $\mathrm{G}$. Insulin resistance and hypersecretion in obesity. J Clin Invest 1997;100:1166-73.

5. Lakka HM, Laaksonen DE, Lakka TA, Niskanem LK, Kumpusalo E, Tuomilehto J, et al. The metabolic syndrome and total and cardiovascular disease mortality in middle-aged men. JAMA 2002;288:2709-16.

6. Ford ES, Giles WH. A comparison of the prevalence of the metabolic syndrome using two proposed definitions. Diabetes Care 2003:26:575-81.

7. Haffner S, Taegtmeyer H. Epidemic obesity and the metabolic syndrome. Circulation 2003;108:1541-5.

8. Gang H, Qiao Q, Tuomilehto J, Balkau B, Borchjohnsen K, Pyorala K;for DECODE Study Group. Prevalence of the metabolic syndrome and its relation to all-cause and cardiovascular mortality in nondiabetic European men and women. Arch Intern Med 2004;164:1066-76.

9. Girman CJ, Rhodes T, Mercuri M, Pyörälä K, Kjekshus J, Pedersen TR, et al;for the 4S Group and AFCAPS/TextCAPS Research Group. The metabolic syndrome and risk of major coronary events in the Scandinavian simvastatin survival study (4S) and the air force/Texas coronary atherosclerosis prevention study (AFCAPS/TextCAPS). Am J Cardiol 2004;93:136-41.

10. Lee WJ, Huang MT, Wang W, Lin CM, Chen TC, Lai IR. Effects of obesity surgery on the metabolic syndrome. Arch Surg 2004; 139(10):1088-92.

11. Case CC, Jones PH, Nelson K, O'Brian Smith E, Ballantyne $\mathrm{CM}$. Impact of weight loss on the metabolic syndrome. Diabetes Obes Metab 2002;4:407-14.

12. NIH Conference: gastrointestinal surgery for severe obesity. Ann Intern Med 1991;115:959-61.

13. Brolin RE. Bariatric surgery and long-term control of morbid obesity. JAMA 2002;288:2793-6.

14. Mun EC, Blackburn GL, Matthews JB. Current status of medical and surgical therapy for obesity. Gastroenterology 2001;120:669-81.

15. Jones Jr KB. Experience with the Roux-en-Y gastric by-pass, and commentary on current trends. Obes Surg 2000;10: 183-5.

16. Brolin RE, Kenler HA, Wilson AC, Kuo PT, Cody RP. Serum lipids after gastric bypass surgery for morbid obesity. Int J Obes Relat Metab Disord 1990;14:939-50.

17. Wolf AM, Beisegel U, Kortner B, Kuhlman HW. Does gastric restriction surgery reduce the risks of metabolic diseases? Obes Surg 1998;8:9-13.

18. Brolin RE. Update. NIH Consensus Conference. Gastrointestinal surgery for severe obesity. Nutrition 1996;12:403-4.

19. Grace DM. Gastric reduction procedures for treating severe obesity. Am J Clin Nutr 1992;55:S556-9.

20. Duncan BB, Schmidt MI, Chambless LE, Folsom AR, Carpenter M, Heiss G. Fibrinogen, other putative markers of inflammation, and weight gain in middled-aged adults - The ARIC Study. Obes Res 2000;8(4):279-86.
21. Pratley R, Wilson C, Bogardus C. Relation of white blood cell count to obesity and insulin resistance: effect of race and gender. Obes Res 1995;3:563-71.

22. Yarnell JWG, Baker IA, Sweetnam PA. Fibrinogen, viscosity, and white blood cell count are major risk factors for ischemic heart disease: the Caerphilly and Speedwell collaborative heart disease studies. Circulation 1999;83:836-44.

23. Schmidt MI, Duncan BB, Sharrett AR, Lindberg G, Savage PJ, Offenbacher $\mathrm{S}$, et al. Markers of inflammation and prediction of diabetes mellitus in adults (Atherosclerosis Risk Communities Study): a cohort study. Lancet 1999;353(9165):1649-52.

24. WHO. Obesity preventing and managing the global epidemic, 1998.

25. Kolanowski J. Surgical treatment for morbid obesity. Br Med Bull 1977;53:433-4.

26. McTigue KM, Harris R, Hemphill B, Lux L, Sutton S, Bunton AJ, et al. Screening and interventions for obesity in adults: summary of the evidence for us prevention services task force. Ann Intern Med 2003;139(11):933-49.

27. Pories WJ, Swanson MS, MacDonald KG, Long SB, Morris PG, Brown BM, et al. Who would have thought? An operation proves to be the most effective therapy for adult onset diabetes mellitus. Ann Surg 1995;222:339-52.

28. Schauer PR, Burguera B, Ikramuddin S, Cottam D, Gourash W, Hamad G, et al. Effect of laparoscopic Roux-en-Y gastric bypass on type 2 diabetes mellitus. Ann Surg 2003;238 (4):467-84.

29. Sugerman HJ, Wolfe LG, Sica DA, Clore JN. Diabetes and hypertension in severe obesity and effects of gastric bypassinduced weight loss. Ann Surg 2003;237(6):751-6.

30. Wittgrove AC, Clark GW. Laparoscopic gastric bypass Rouxen-Y-500 patients: technique and results, with 3-60 months follow-up. Obes Surg 2000;10(3):233-9.

31. Schauer PR, Ikramuddin S, Gourash W, Ramanathan R, Luketich J. Outcomes after laparoscopic Roux-en-Y gastric bypass for morbid obesity. Ann Surg 2000;232(4):515-29.

32. Cummings DE, Weigle DS, Frayo RS, Breen PA, Ma MK, Dellinger EP, et al. Human plasma ghrelin levels after dietinduced weight loss and gastric bypass surgery. N Engl J Med 2002;346(21):1623-30.

33. Zander M, Madsbad S, Madsen JL, Holst JJ. Effect of 6-week course of glucagon-like peptide-1 on glycemic control, insulin sensitivity, and $\beta$-cell function in type 2 diabetes: a parallelgroup study. Lancet 2002;359:824-30.

34. Campbell PJ, Carlson MG, Nurjhan N. Fat metabolism in human obesity. Am J Physiol 1994;266:E600.

35. Lewis GF, Uffelman KD, Szeto LW, Steiner G. Effects of acute hiperinsulinemia on VLDL triglyceride and VLDL ApoB production in normal weight and obese individuals. Diabetes 1993;42(6):833-42.

36. Malmstrom R, Packard CJ, Caslake M, Bedford D, Stewart $P$, Yki-Jarvinen $\mathrm{H}$, et al. Defective regulation of triglyceride metabolism by insulin in the liver in NIDDM. Diabetologia 1997;40(4):454-62.

37. Swenson TL. The role of the cholesteryl ester transfer protein in lipoprotein metabolism. Diabetes Metab Rev $1991 ; 7(3): 139-53$

38. Ferrannini E, Buzzigoli G, Bonadonna R, Giorico MA, Oleggini $M$, Graziadei $L$, et al. Insulin resistance in essential hypertension. N Engl J Med 1987;317(6):350-7.

39. Swislocki AL, Hoffman BB, Reaven GM. Insulin resistance, glucose intolerance and hyperinsulinemia in patients with hypertension. Am J Hypertens 1989;2(6 pt. 1):419-23.

40. Muscelli E, Natali A, Bianchi S, Bigazzi R, Galvan AQ, Sironi $A M$, et al. Effect of insulin on renal sodium and uric acid handling in essential hypertension. Am $\mathbf{J}$ Hypertens 1996;9(8):746-52.

41. Reaven GM, Lithell H, Landsberg L. Hypertension and associated metabolic abnormalities - the role of insulin resistance and the sympathoadrenal system. N Engl J Med 1996;334:374-82.

42. Stuhlinger MC, Abbasi F, Chu JW, Lamendola C, McLaughlin $\mathrm{TL}$, Cooke JP, et al. Relationship between insulin resistance and an endogenous nitric oxide synthase inhibitor. JAMA 2002;287(11):1420-6. 
43. Visser M, Bouter LM, McQuillan GM, Wener MH, Harris TB. Elevated C-reactive protein levels in overweight and obese adults. JAMA 1999;282:2131-5.

44. Nakanishi N, Sato M, Shirai K, Nakajima K, Murakami S, Takatorige T, et al. Association between white blood cell count and features of the metabolic syndrome in Japanese male office workers. Ind Health 2002;40(3):273-7.

45. Alvarez-Cordero R. Final reflexions: wellness after obesity surgery. World J Surg 1998;22:1018-21.

46. Sjöström L, Lindroos AK, Peltonen M, Torgerson J, Bouchard C, Carlsson B, et al. Lifestyle, diabetes, and cardiovascular risk factors 10 years after bariatric surgery. N Engl J Med 2004;351(26):2683-93.
Endereço para correspondência:

Perseu Seixas de Carvalho

Rua Eurico Aguiar 888, salas 905/906

29055-280 Vitória, ES

Fax: (27) 3324-6498

E-mail: perseu@npd.ufes.br 